\title{
A Tool to Predict the Probability of Summer Severe Weather in East-Central Florida
}

\author{
WILLIAM H. BAUMAN III \\ NASA Applied Meteorology Unit / ENSCO, Inc., Cape Canaveral Air Force Station, Florida \\ WILLIAM P. ROEDER \\ USAF 45th Weather Squadron, Patrick Air Force Base, Florida
}

(Manuscript received 7 November 2013; review completed 11 March 2014)

\begin{abstract}
People and property at Kennedy Space Center and Cape Canaveral Air Force Station (CCAFS) are at risk from severe weather. Strong winds, hail, and tornadoes can injure individuals and cause costly damage to structures if not properly protected. This is important because central Florida is the thunderstorm capital of the United States and has the highest tornado density in the country, albeit a smaller percentage than the national average of these tornadoes is of significant intensity. The 45th Weather Squadron (45WS) severe weather forecasts are used by managers to determine if they need to schedule activities (such as working on gantries) or protect property (such as a vehicle on a pad). Missed desired lead-time and false-alarm rate statistics show that severe weather in east-central Florida is difficult to forecast during the warm season (May-September). The 45WS tasked the Applied Meteorology Unit (AMU) to develop a locally tuned tool to predict the probability of warm-season severe weather using a 24-yr climatological database from the 1500 UTC (1100 EDT) CCAFS radiosondes (KXMR). The results indicate the proper use of certain stability indices and parameters based on the late-morning KXMR soundings can improve the forecaster's ability to identify days with a severe weather threat. The AMU calculated a total severe potential (TSP) by integrating the important parameters for every 1500 UTC sounding and other factors in the 24-yr database and compared the TSP to reported severe weather occurrences on each day with a sounding. Logistic regression was used to convert the TSPs to probability of severe weather as a first guess tool for the 45WS forecasters.
\end{abstract}

\section{Introduction}

The Applied Meteorology Unit (AMU) is a research-to-operations organization whose purpose is to improve weather support to America's space program at Kennedy Space Center (KSC) and Cape Canaveral Air Force Station (CCAFS), Florida; Vandenberg Air Force Base, California; and Wallops Flight Facility, Virginia (Bauman et al. 2004; Madura et al. 2011). The AMU originally developed a severe weather forecast decision aid (Bauman et al. 2005) based on the 1000 UTC (0600 local time) CCAFS sounding (KXMR). This first-guess tool supplemented existing products used by the forecasters to determine the severe weather threat for the day at KSC, CCAFS, and Patrick Air Force Base (PAFB), Florida. As dictated by Air Force Weather directive, the 45th Weather Squadron (45WS) issues severe weather watches for tornadoes, convective surface winds $\geq 26$ $\mathrm{m} \mathrm{s}^{-1}$ ( $\left.\geq 50 \mathrm{kt}\right)$, and hail $\geq 1.91 \mathrm{~cm}(\geq 0.75 \mathrm{in})$ for KSC, CCAFS, and PAFB. The 45WS forecasters tasked the AMU to develop a locally tuned, first-guess tool because experience had shown them that existing national-scale products are not designed for the typically small-scale, short-lived, isolated severe weather in the east-central Florida warm season.

The original decision aid used a Hyper-Text Markup Language graphical user interface (GUI), and the period of record (POR) included the warm-season months of May-September for the years 1989-2003. The decision aid was modified for use with a Meteorological Interactive Data Display System [MIDDS, based on McIDAS-X software (Space Science and Engineering Center 2012)] GUI in 2009 (Wheeler 2009), and then data from the 2004 to 2009 warm sea- 
sons were added in 2010 (Wheeler 2010). In 2011, data from the 2010 warm season were added to the decision aid, verification statistics were calculated for the total severe potential (TSP), and logistic regression analysis was performed on the 22-yr severe weather database (Watson 2011). The 2011 results indicated that the logistic regression equation did not show an increase in skill over the previously developed TSP. Therefore, the only change to the version of the decision aid developed in Wheeler (2010) was the inclusion of 2010 data in the database.

The new tool reported in this work includes the warm-season months in the $24 \mathrm{yr}$ from 1989 to 2012 and was based on the 1500 UTC (1100 EDT) KXMR sounding instead of the 1000 UTC KXMR sounding; it also builds upon the previous work conducted to develop the 1000 UTC sounding-based tool. The 1500 UTC sounding was used because it is closer in time to when the 45WS usually decides to issue their severe weather watches and should provide better skill than the 1000 UTC sounding. The benefits of the 1500 UTC sounding include showing how the boundary layer has modified in the $3 \mathrm{~h}$ since sunrise, in addition to simply being closer in time to the potential severe weather. The AMU took advantage of using some of the existing climatological databases and methods described in the previously referenced reports to create this tool. Besides using the late-morning soundings for this work, the AMU eliminated $83 \%$ of the subjective questions posed to the forecasters in the previous GUI, thereby streamlining the process of running the tool in MIDDS and creating a more objective assessment of the daily warm-season severe weather threat. The AMU discovered that the subjectivity in the previous GUI sometimes resulted in different severe weather threat assessments for the same day when used by different forecasters. Also, the AMU's statistical analysis determined that some of the parameters were not relevant when considering the severe weather threat. For example, on $95 \%$ of the days with reported severe weather, no severe weather was reported on the previous day. Therefore the questions about persistence in the previous GUI were eliminated. Table 1 provides a summary of each question from the previous GUI, whether or not the question was eliminated in the new 1500 UTC GUI, and the reason why the question was removed or remained. This tool only indicates if the general conditions are conducive to severe weather. The actual location and timing of the severe weather will be influenced strongly by lowlevel boundary interactions, such as sea breeze fronts from the Atlantic Ocean and/or Gulf of Mexico and Indian River, and/or Banana River breeze fronts, thunderstorm outflows, and other sources. This tool, being based primarily on the KXMR sounding, does not account for those crucial low-level boundary interacttions.

\section{Data and methods}

The AMU had compiled three existing datasets during previous work that were used in this task after they were updated with 2011 and 2012 data. They included upper-level (200 hPa) jet stream analyses, severe storm reports, and daily flow regimes. The two new datasets required for this task were the 1500 UTC KXMR soundings and the stability parameters derived from those soundings.

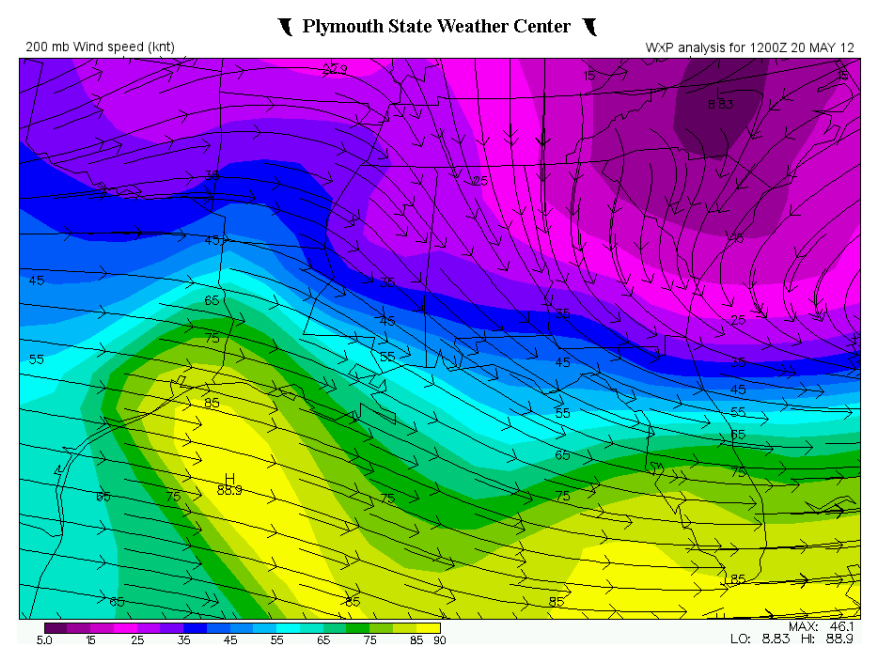

Figure 1. Example of a 200-hPa wind and streamline map generated from the PSU Weather Center used in this work to identify the jet stream position. Lines with arrows indicate the wind direction, and the shaded regions show the wind speed in kt. Click image for an external version; this applies to all figures hereafter.

\section{a. Existing datasets}

To update the existing datasets, the AMU generated and downloaded the 200-hPa wind and streamline maps (e.g., Fig. 1) from the Plymouth State University (PSU) Weather Center (vortex.plymouth.edu/u-make. html) for the 2011 and 2012 warm seasons. The maps were analyzed subjectively to visually determine the jet stream position, and the results were entered into the existing 1989-2010 AMU jet-stream analysis database. Next, the AMU downloaded the 2011 and 2012 warm-season severe storm reports from the National Climatic Data Center Storm Events Database (www.ncdc.noaa.gov/stormevents) and then added the 
Table 1. Summary of the parameters from the previous severe tool GUI that were eliminated or carried over into the new 1500 UTC GUI.

\begin{tabular}{|c|c|c|}
\hline GUI Question & Eliminated? & Reason \\
\hline Is severe weather mentioned in the FXUS62 bulletin? & Yes & $\begin{array}{l}\text { Forecasters sometimes inferred severe weather from other terms that were } \\
\text { used, such as "strong." }\end{array}$ \\
\hline Was severe weather mentioned in the previous bulletin? & Yes & The previous bulletin was not relevant for the current day. \\
\hline Was severe weather reported by Tampa or Melbourne? & Yes & Persistence was not relevant $95 \%$ of the time. \\
\hline $\begin{array}{l}\text { Was severe weather reported by Tallahassee or } \\
\text { Jacksonville? }\end{array}$ & Yes & $\begin{array}{l}\text { Persistence was not relevant } 95 \% \text { of the time, and these reports were } \\
\text { geographically irrelevant. }\end{array}$ \\
\hline $\begin{array}{l}\text { Is there a front/squall line in northwestern FL moving to } \\
\text { the southeast? }\end{array}$ & Yes & $\begin{array}{l}\text { Forecasters did not always agree on the answer to this question- too } \\
\text { subjective. }\end{array}$ \\
\hline Is there a distinct moist/dry boundary across central FL? & Yes & $\begin{array}{l}\text { Forecasters did not always agree on the answer to this question- too } \\
\text { subjective. }\end{array}$ \\
\hline $\begin{array}{l}\text { Do the sounding winds veer with height from surface to } \\
3048 \mathrm{~m}(10000 \mathrm{ft}) \text { ? }\end{array}$ & Yes & $\begin{array}{l}\text { On days with reported severe weather, the winds were veering } 40 \% \text { of the } \\
\text { time and backing } 60 \% \text { of the time. }\end{array}$ \\
\hline $\begin{array}{l}\text { Is there a } 200-\mathrm{hPa} \text { speed max, right-entrance region, left- } \\
\text { exit region, or other upper-level divergence nearby? }\end{array}$ & No & $\begin{array}{l}\text { The position of a } 200-\mathrm{hPa} \text { speed max was directly related to days with } \\
\text { reported severe weather. }\end{array}$ \\
\hline What is the flow regime? & No & $\begin{array}{l}\text { The flow regime was related directly to days with reported severe } \\
\text { weather. }\end{array}$ \\
\hline If a sea breeze forms will it stay east of I-95? & Yes & $\begin{array}{l}\text { Although relevant, it could not be quantified objectively. The new GUI } \\
\text { has a statement reminding the forecasters to consider this parameter. }\end{array}$ \\
\hline Are you forecasting a late developing sea breeze? & Yes & $\begin{array}{l}\text { Although relevant, it could not be quantified objectively. The new GUI } \\
\text { has a statement reminding the forecasters to consider this parameter. }\end{array}$ \\
\hline $\begin{array}{l}\text { Are you forecasting or observing multiple boundary } \\
\text { collisions? }\end{array}$ & Yes & $\begin{array}{l}\text { Although relevant, it could not be quantified objectively. The new GUI } \\
\text { has a statement reminding the forecasters to consider this parameter. }\end{array}$ \\
\hline
\end{tabular}

severe events for Brevard, Volusia, Indian River, Seminole, Osceola, and Orange Counties in Florida (Fig. 2) to the existing 1989-2010 AMU severe-storm reports database. Severe weather events included tornadoes, waterspouts, convective surface winds $\geq 26 \mathrm{~m}$ $\mathrm{s}^{-1}(\geq 50 \mathrm{kt})$ and/or hail with a diameter $\geq 1.91 \mathrm{~cm}$ ( $\geq 0.75$ in) through 2009 and $\geq 2.54 \mathrm{~cm} \mathrm{(} \geq 1.00$ in) after 2009. Although KSC/CCAFS/PAFB are all located in Brevard County, the other inland and coastal counties that surround or are near Brevard County were needed to make sure the database had enough events to derive meaningful statistical relationships. In addition, even though these severe events may not have occurred at KSC/CCAFS/PAFB, possible impacts to operations needed to be tracked if severe weather was occurring in relatively close proximity to the space center. Finally, the main triggers of convection in the warm season are the location, movement, and strength of the local sea breeze front and collisions with other lowlevel boundaries, such as thunderstorm outflows. As a proxy for these boundaries, the AMU added the 2011 and 2012 daily lightning flow regimes (Lericos et al. 2002; Lambert and Roeder 2008) to the 1989-2010 AMU flow regime database. These flow regimes are based on the mean wind direction in the 1000-700-hPa layer, which represents an optimum compromise be- tween the lower-altitude flow that governs the location and inland speed of the sea breeze front and the higher altitude that steers thunderstorms.

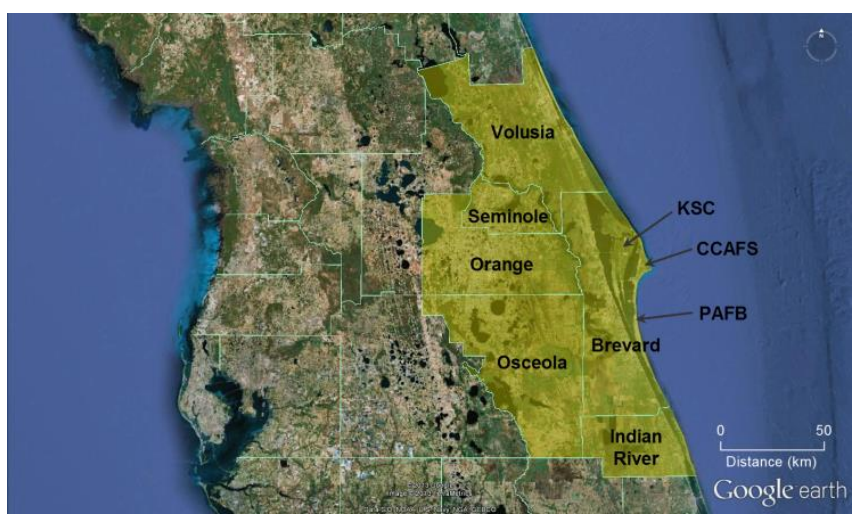

Figure 2. Map of central FL showing the six counties (shaded in yellow) included in the severe weather events database. The location of KSC, CCAFS, and PAFB are shown on the map; all three are in Brevard County.

\section{b. New datasets}

The AMU received sounding observations in the form of ASCII text files from the contractor (Computer Sciences Raytheon) who operates the KXMR sounding site. The AMU selected soundings with release times between 1430 and 1530 UTC and quality 
controlled each sounding. If more than one sounding on a single day was available between 1430 and 1530 UTC, the sounding closest in time to 1500 UTC or the one that was most complete was selected. Each sounding contained separate mandatory, significant, and 304.8-m (1000-ft) levels, which the AMU merged to create one complete file for each sounding.

Warm-season soundings over 24 yr resulted in a total of 2842 days with one sounding released between 1430 and 1530 UTC out of a possible 3672. The AMU removed 14 soundings from the database on days when KSC/CCAFS/PAFB was under the influence of a tropical cyclone because these did not represent the days where the convection is dominated by local effects, as required for this tool. Another 30 soundings failed the quality control checks owing to missing data or physically impossible values, and were excluded from the database. After these removals, a total of 2798 soundings were available for developing the severe weather tool (SWT). The following 24 severe weather indices and parameters were generated from the soundings using the default settings in McIDAS-X software (Space Science and Engineering Center 2012):

- lifted index (LI)

- $\mathrm{K}$-index (KI)

- Thompson index (TI)

- Showalter stability index (SSI)

- total totals (TT)

- cross totals (CT)

- vertical totals (VT)

- $\quad$ severe weather threat index (SWEAT)

- convective available potential energy (CAPE)

- CAPE based on the maximum equivalent potential temperature (CAPE Max $\theta_{e}$ )

- CAPE based on the forecast maximum temperature (CAPE FMaxT)

- convective inhibition (CIN)

- precipitable water $(\mathrm{PW})$

- temperature at $850 \mathrm{hPa}(\mathrm{T} 850)$

- temperature at $500 \mathrm{hPa}$ (T500)

- average relative humidity in the $1000-700-\mathrm{hPa}$ layer (Avg70RH)

- average relative humidity in the $850-500-\mathrm{hPa}$ layer (Avg85RH)

- average relative humidity in the $850-600-\mathrm{hPa}$ layer (Avg86RH)

- microburst day potential index (MDPI; Wheeler and Roeder 1996)
- inversion height below $2438 \mathrm{~m}(8000 \mathrm{ft})$

- $\quad$ wind speed $\geq 12.8 \mathrm{~m} \mathrm{~s}^{-1}$ ( $\geq 25 \mathrm{kt}$ ) and wind direction $\geq 109^{\circ}$ and $\leq 270^{\circ}$ at $850 \mathrm{hPa}$ (850 Jet)

- veering winds from the surface to $3048 \mathrm{~m} \mathrm{(10000}$ ft) (WarmAdv)

- $\quad$ storm-relative helicity

- storm-relative motion (speed and direction)

\section{Analysis and results}

The analysis and results are described in three sections. The first section shows how the climatological stability indices and parameters were analyzed, leading to the development of severe weather threat scores and a TSP for each day in the POR. The second section shows how the TSP values were assessed to develop a best-fit logistic regression curve based on the distribution of reported severe weather. The third and final section discusses the (i) development of the GUI in the 45WS MIDDS and (ii) transition of the GUI to operations.

\section{a. Stability thresholds and threat scores}

After generating the stability indices and parameters, the AMU categorized the days with reported severe weather and days without reported severe weather by threshold values for each index and then developed charts showing the percent of time severe weather was reported based on specific thresholds. The thresholds were the same as those used in the original severe weather decision aid (Bauman et al. 2005). An example using TT is shown in Fig. 3. When the TT was in the low category (TT $\leq 45)$, severe weather was reported $11 \%$ of the time. When TT was in the medium category $(46 \leq \mathrm{TT} \leq 48)$, severe weather was reported $25 \%$ of the time. When TT was in the high category (TT >48), severe weather was reported $45 \%$ of the time.

The AMU used the categorized thresholds from each index to determine which would be useful predictors of severe weather occurrence. A threat score for each index was derived from the percent of time severe weather occurred in each threshold category and then was scaled between 0 and 10 by dividing the percent value by 10 . Based on this method, the TT threat scores for the low, medium, and high threshold categories were $1.1,2.5$, and 4.5 , respectively. The AMU used these scaled threat score values as the basis to compute the TSP from multiple indices and parameters. 


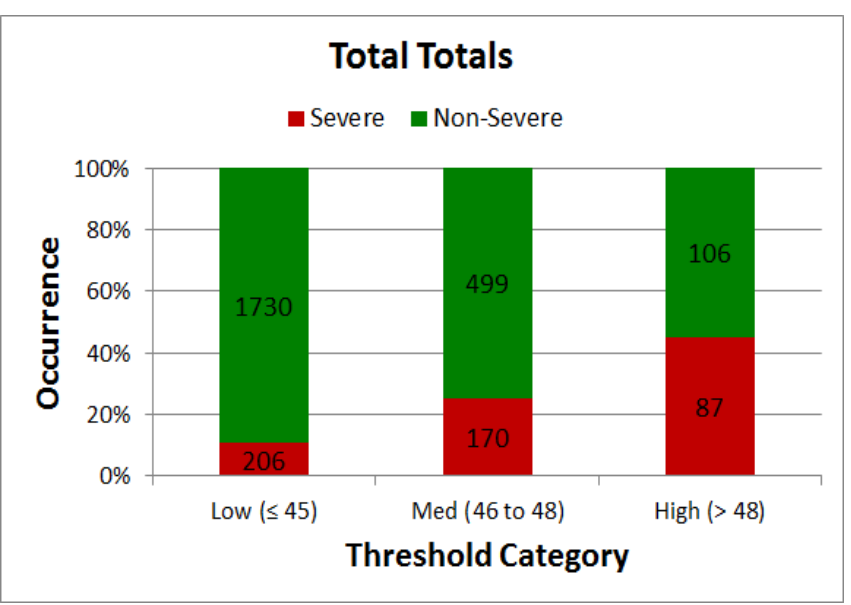

Figure 3. Stacked bar chart of TT for the low, medium, and high threshold categories showing percent occurrence of the number of days with reported severe weather (red) and days with no reported severe weather (green).

Figure 4 compares the threat score for each stability index in each category. Lines with steeper slopes show a correlation to reported severe weather by having low threat scores in the low category increasing markedly to higher threat scores in the high or very high categories. Based on the slope of each line in Fig. 4 , the best stability index indicators of severe weather occurrence were SSI, TT, SWEAT, LI, and VT, as they had the largest increase in severe weather threat score from lowest to highest threshold category. The CT, TI, and KI slopes were not as steep, representing a smaller threat score change across the threshold categories. Therefore, they were not as good as the other indices in separating the likelihood of severe weather occurrence based on the set of thresholds. Similarly, the thresholds of energy indices derived from the soundings are shown in Fig. 5. The CIN, CAPE Max $\theta_{e}$, and CAPE FMaxT were the best energy index indicators with slopes similar to the CT, TI, and KI stability indices. Storm-relative helicity-not shown in the charts - was not incorporated into the tool because its threat score decreased across the low, medium, and high categories.

The AMU also considered moisture parameters derived from the soundings as severe weather indicators, and the resulting chart is shown in Fig. 6. The values of the Avg85RH and Avg86RH increase from the low to medium threshold categories but then decrease at the high threshold category, indicating they are poor predictors so were not used in the tool. The Avg70RH and PW both increased across the threshold categories and were used as predictors. Some other parameters considered as possible indicators of severe

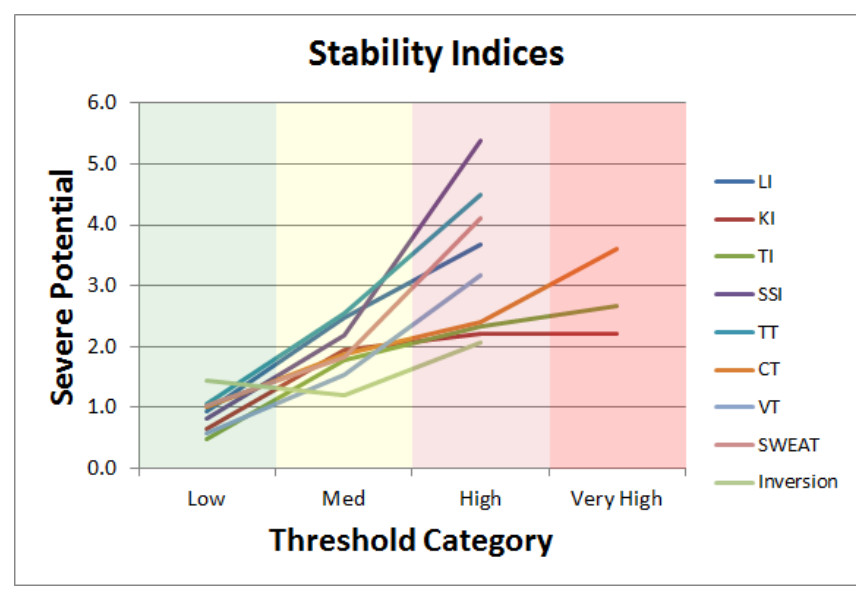

Figure 4. Line chart of stability indices showing the threat score for each index in each threshold category.

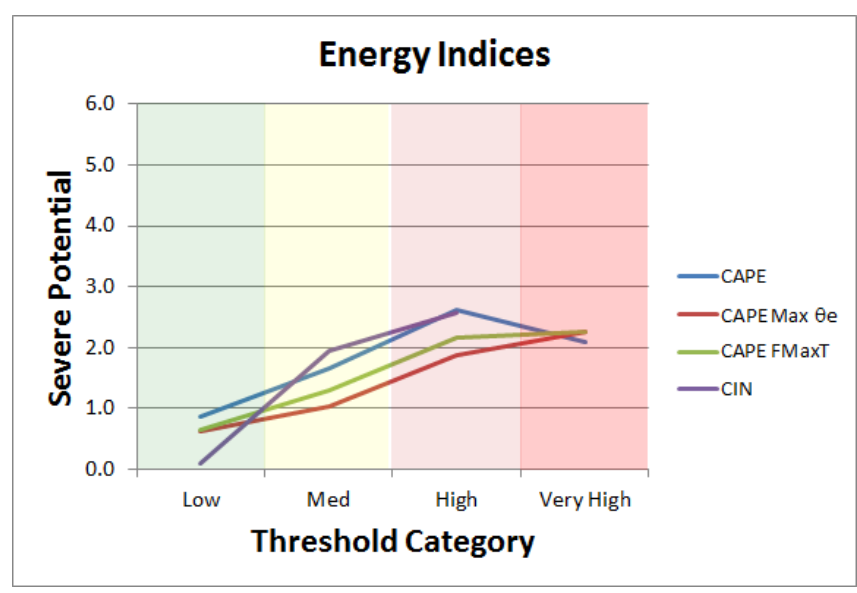

Figure 5. Same as Fig. 4 except for energy indices.

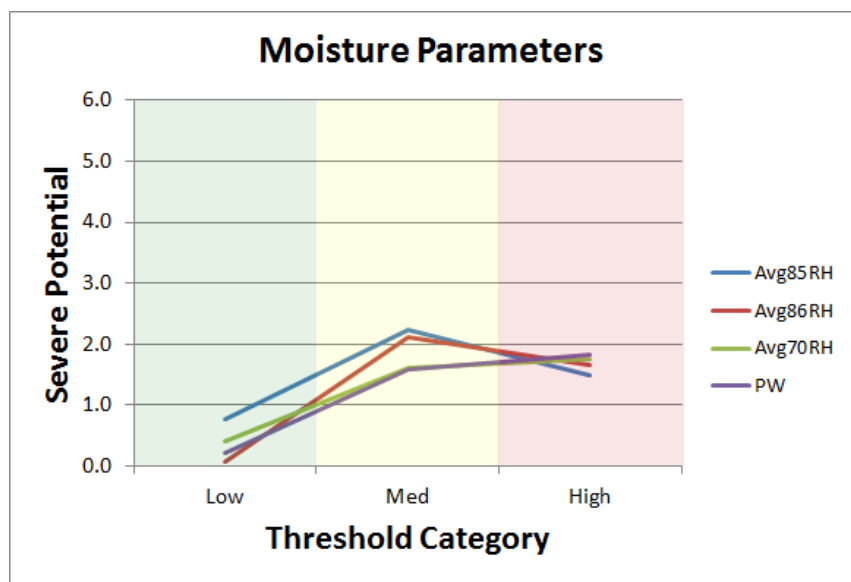

Figure 6. Same as Fig. 4 except for moisture parameters.

weather are shown in Fig. 7. They include T850, MDPI, 850 Jet, WarmAdv, and T500. Of these parameters, only the 850 Jet showed a significant enough correlation to reported severe weather that it was incorporated into the tool. 


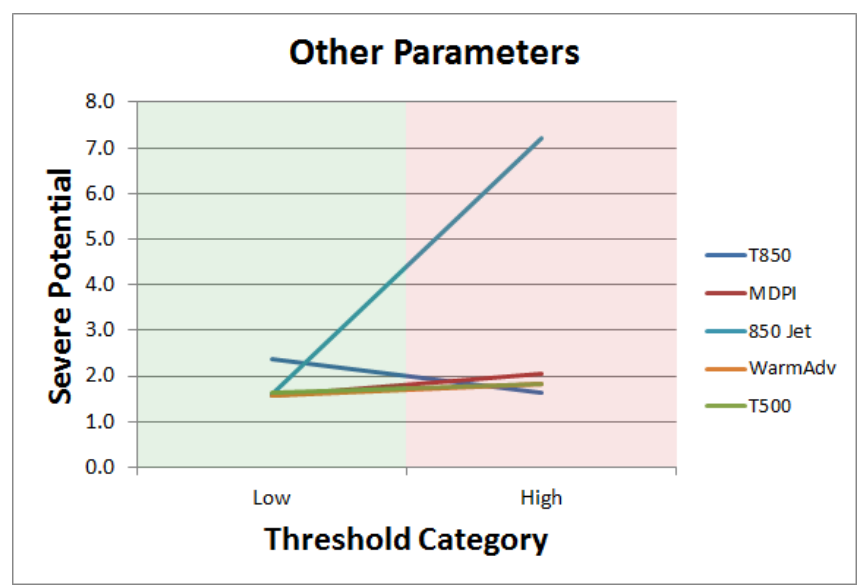

Figure 7. Line chart of other parameters showing the threat score for each parameter in two threshold categories. These parameters are binary, so only two categories are possible, unlike the 3-4 categories used for the other parameters, which are continuous.

Finally, the AMU considered two other parameters known to contribute to severe weather potential: the peninsular lightning flow regime (Lericos et al. 2002) and the 200-hPa jet position (Uccellini and Johnson 1979). Previous research indicated the type of flow regime had some influence on the frequency and intensity of convective winds (Ander et al. 2009). The threat scores for each of the flow regimes are shown in Fig. 8. The two westerly regimes-northwest (NW) and southwest (SW)-result in the highest threat scores because those regimes favor thunderstorm frequency in eastern Florida. In addition, these thunderstorms tend to be more intense because they have gained strength from ground heating throughout the day and from boundary interactions as they approach the sea breeze front from the Atlantic Ocean and other low-level boundaries in the eastern half of the Florida peninsula. The highest threat scores based on the 200hPa flow and jet position (Fig. 9), relative to eastcentral Florida, occur under the influence of left-exit and right-entrance regions (Uccellini and Johnson 1979) and in other regions of upper-level divergence.

The AMU compiled the results of all parameters' threat scores for each sounding, flow regime, and 200$\mathrm{hPa}$ jet position for the 24-yr database in two Excel spreadsheets. One spreadsheet contained the threat scores for each day with reported severe weather and the other spreadsheet had days with no reported severe weather. Figure 10 shows a sample of the spreadsheet with threat scores for days with reported severe weather. The daily TSP was determined by summing the individual threat scores from each parameter in each row. On days with reported severe weather, the TSP

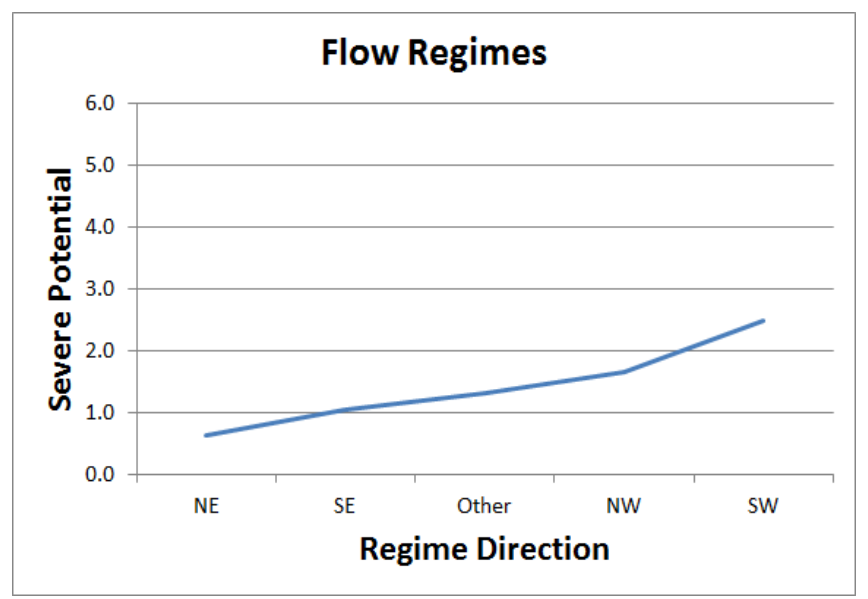

Figure 8. Line chart of flow regimes and corresponding threat scores.

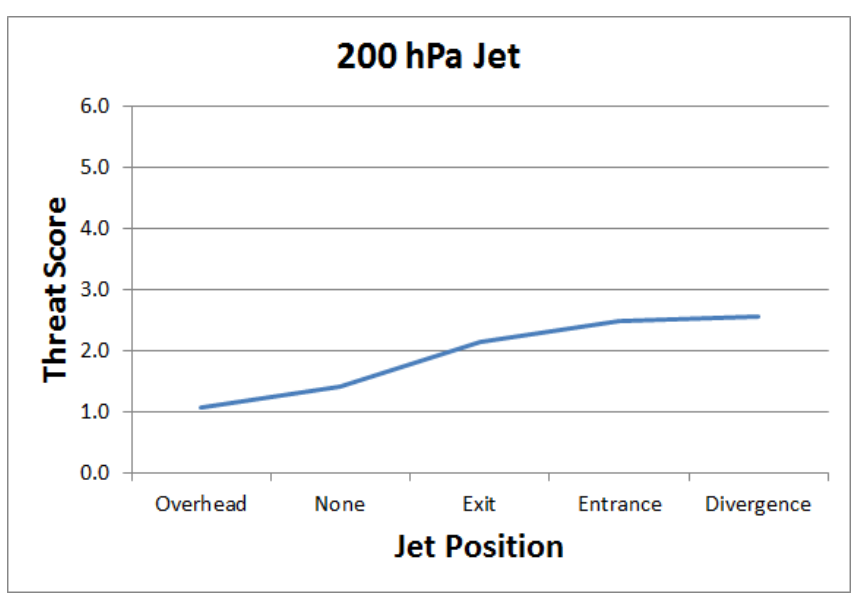

Figure 9. Same as Fig. 8 except for the 200-hPa jet.

ranged from 15 to 50 with a median of 30 . On days with no reported severe weather, the TSP ranged from 12 to 41 with a median of 22 .

The TSP distribution for days with reported severe weather and for days with no reported severe weather is an important factor in the development of this tool and should demonstrate the ability of the TSP to indicate the severe weather potential. Figure 11 shows the distributions of days with and without reported severe weather. Although there is some overlap, the frequency maxima of the distributions are distinct, indicating the TSP distribution provides insight into the severe weather potential. On days when severe weather was reported, the TSP was $\geq 30$ during $50 \%$ of those days. On days with no reported severe weather, the TSP was $\geq 30$ during $6 \%$ of those days. Conversely, when the TSP was $\leq 24,69 \%$ of the days had no reported severe weather, while $15 \%$ of days had reported severe weather. 


\begin{tabular}{|c|c|c|c|c|c|c|c|c|c|c|c|c|c|c|c|c|c|c|c|c|}
\hline \multicolumn{3}{|c|}{ Date } & \multicolumn{16}{|c|}{ Stability and Moisture Severe Potential } & \multirow{2}{*}{$\frac{\text { Raw }}{\text { TTS }}$} & \multirow{2}{*}{$\begin{array}{c}\text { Rounded } \\
\text { TTS }\end{array}$} \\
\hline Year & Month & Day & $\mathrm{LI}$ & $\mathrm{KI}$ & $\mathrm{TI}$ & SSI & $\pi$ & CT & VT & PW & SWEAT & CAPE FMaxT & CIN & Flow Regime & Jet Position & LLJet & MDPI & RH700 & & \\
\hline 1989 & MAY & 1 & 0.9 & 0.7 & 0.5 & 0.8 & 1.1 & 1.0 & 3.2 & 1.7 & 1.0 & 0.6 & 2.6 & 2.5 & 2.6 & 1.6 & 1.6 & 1.7 & 24.1 & 24 \\
\hline 1989 & AUG & 9 & 0.9 & 0.7 & 0.5 & 0.8 & 1.1 & 1.0 & 1.5 & 1.7 & 1.8 & 2.2 & & 2.5 & 2.1 & 1.6 & 1.6 & 1.7 & 21.7 & 22 \\
\hline 1989 & AUG & 10 & 2.5 & 0.7 & 1.8 & 0.8 & 1.1 & 1.0 & 0.6 & 1.7 & 1.0 & 2.2 & 1.0 & 2.5 & 2.1 & 1.6 & 1.6 & 1.7 & 23.9 & 24 \\
\hline 1990 & JUN & 26 & 2.5 & 2.2 & 2.7 & 2.2 & 2.5 & 2.4 & 3.2 & 1.7 & 1.8 & 2.3 & & 1.1 & 2.1 & 1.6 & 1.6 & 1.7 & 31.6 & 32 \\
\hline 1990 & JUL & 2 & 3.7 & 2.2 & 2.7 & 5.4 & 4.5 & 3.6 & 3.2 & 1.7 & 1.8 & 2.3 & & 1.7 & 2.6 & 1.6 & 1.6 & 1.7 & 40.3 & 40 \\
\hline 1990 & JUL & 12 & 2.5 & 2.2 & 2.3 & 2.2 & 2.5 & 2.4 & 3.2 & 1.7 & 1.8 & 2.2 & & 1.1 & 2.6 & 1.6 & 1.6 & 1.7 & 31.6 & 32 \\
\hline 1990 & JUL & 22 & 2.5 & 2.2 & 2.3 & 2.2 & 2.5 & 2.4 & 1.5 & 1.7 & 1.8 & 2.3 & 1.0 & 2.5 & 2.6 & 1.6 & 1.6 & 1.7 & 32.4 & 32 \\
\hline 1990 & JUL & 25 & 2.5 & 2.2 & 2.3 & 2.2 & 2.5 & 1.9 & 3.2 & 1.7 & 1.8 & 2.3 & & 1.3 & 1.4 & 1.6 & 1.6 & 1.7 & 30.2 & 30 \\
\hline 1990 & AUG & 3 & 2.5 & 2.2 & 2.7 & 2.2 & 2.5 & 2.4 & 1.5 & 1.7 & 1.8 & 2.3 & & 2.5 & 1.4 & 1.6 & 1.6 & 1.7 & 30.6 & 31 \\
\hline 1990 & AUG & 9 & 2.5 & 2.2 & 2.3 & 2.2 & 2.5 & 1.9 & 1.5 & 1.7 & 1.8 & 2.3 & & 2.5 & 2.6 & 1.6 & 1.6 & 1.7 & 30.9 & 31 \\
\hline 1990 & AUG & 10 & 0.9 & 2.2 & 1.8 & 0.8 & 1.1 & 1.0 & 1.5 & 1.7 & 1.8 & 1.3 & & 2.5 & 2.1 & 1.6 & 1.6 & 1.7 & 23.6 & 24 \\
\hline 1990 & AUG & 13 & 2.5 & 2.2 & 2.3 & 2.2 & 2.5 & 1.9 & 1.5 & 1.7 & 1.8 & 2.3 & 1.0 & 1.1 & 2.1 & 1.6 & 1.6 & 1.7 & 30.0 & 30 \\
\hline 1991 & MAY & 31 & 2.5 & 2.2 & 1.8 & 2.2 & 4.5 & 1.9 & 3.2 & 1.7 & 1.8 & 1.3 & 2.6 & 1.7 & 2.6 & 1.6 & 1.6 & 1.7 & 34.9 & 35 \\
\hline 1991 & JUN & 14 & 2.5 & 2.2 & 2.7 & 2.2 & 4.5 & 3.6 & 1.5 & 1.7 & 1.8 & 2.2 & & 1.1 & 2.6 & 1.6 & 1.6 & 1.7 & 33.5 & 34 \\
\hline 1991 & JUN & 18 & 2.5 & 2.2 & 2.7 & 2.2 & 2.5 & 3.6 & 1.5 & 1.7 & 1.8 & 2.3 & 1.9 & 2.5 & 2.6 & 1.6 & 1.6 & 1.7 & 34.9 & 35 \\
\hline 1991 & JUL & 10 & 2.5 & 2.2 & 2.3 & 0.8 & 1.1 & 1.0 & 1.5 & 1.7 & 1.8 & 2.3 & 1.9 & 2.5 & 2.6 & 1.6 & 1.6 & 1.7 & 29.1 & 29 \\
\hline 1991 & JUL & 15 & 3.7 & 2.2 & 2.3 & 2.2 & 4.5 & 2.4 & 3.2 & 1.7 & 1.8 & 2.3 & 1.9 & 1.1 & 1.4 & 1.6 & 2.1 & 1.7 & 36.1 & 36 \\
\hline 1991 & JUL & 16 & 3.7 & 2.2 & 2.7 & 5.4 & 4.5 & 3.6 & 3.2 & 1.7 & 1.8 & 2.3 & 1.0 & 2.5 & 2.6 & 1.6 & 2.1 & 1.7 & 42.6 & 43 \\
\hline
\end{tabular}

Figure 10. A portion of the Excel spreadsheet used to calculate the TSP for each day based on the sounding parameters, flow regime, and 200-hPa jet position.

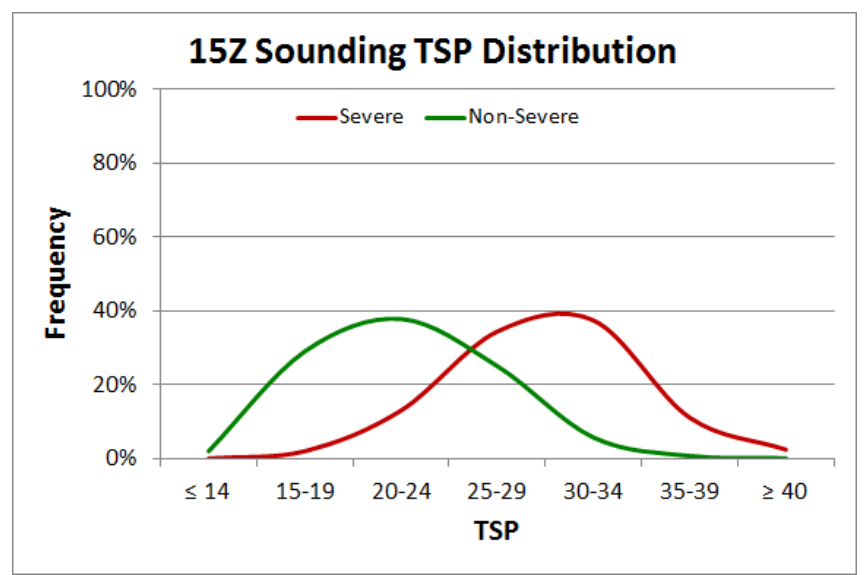

Figure 11. The TSP distribution on days with reported severe weather (red line) and days with no reported severe weather (green line) for the 1500 UTC sounding at CCAFS (i.e., KXMR).

Another consideration for forecasters is the occurrence of reported severe weather based on TSP category. For example, as Fig. 12 illustrates, when the TSP was $\geq 40$, severe weather was reported $92 \%$ of the time. Although that is significant, only $2 \%$ of days with reported severe weather were in this TSP range. So, even though this TSP category does not occur often, when it does occur, severe weather is very likely. This is expected because severe weather events are rare; thus, a good predictor should likewise occur infrequently.

After discussing the TSP categories with the 45WS forecasters, the AMU decided the seven numerical categories may be too broad to provide quality guidance to the forecasters, and thus considered using single TSP values instead of categories to provide higher fidelity output of the frequency of occurrence

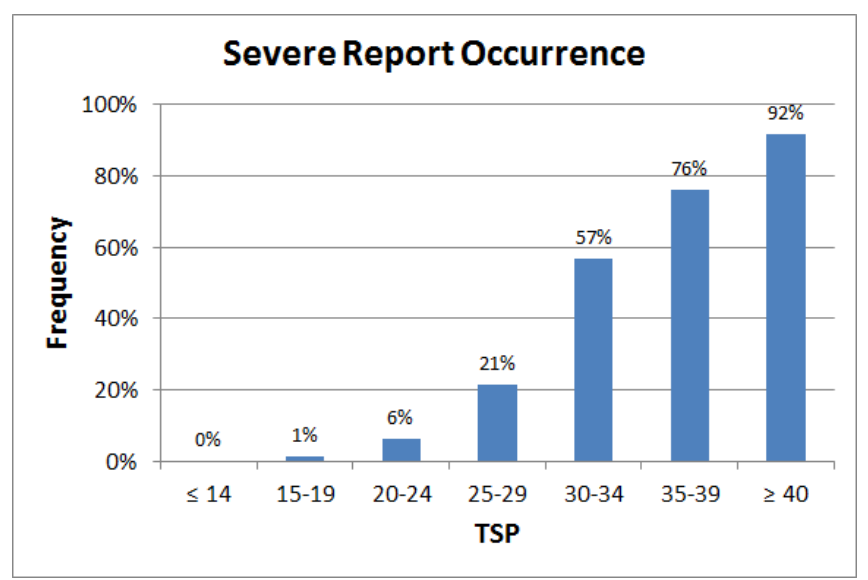

Figure 12. The distribution of reported severe weather frequency based on seven TSP categories.

of reported severe weather. As a result, Fig. 13 shows a line chart of each TSP value where the sudden drop of probability for TSP values between 44 and 49 are likely due to a very small sample size and a large rural area where severe weather would not be reported if it occurred. Although this method provides greater detail, the relationship is more chaotic and prone to more noise than the categorical data-especially at higher TSP values with a smaller sample size.

\section{b. Best-fit logistic regression curve}

To help minimize the noisy behavior and create a more useful tool for the forecasters, the AMU fit several types of curves to the data including logarithmmic and polynomial. A second-order polynomial is shown in Fig. 14. However, the polynomial curve reached a maximum of $59 \%$ at a TSP of 37 and fell below $0 \%$ at a TSP of 18 . Further examination of the 


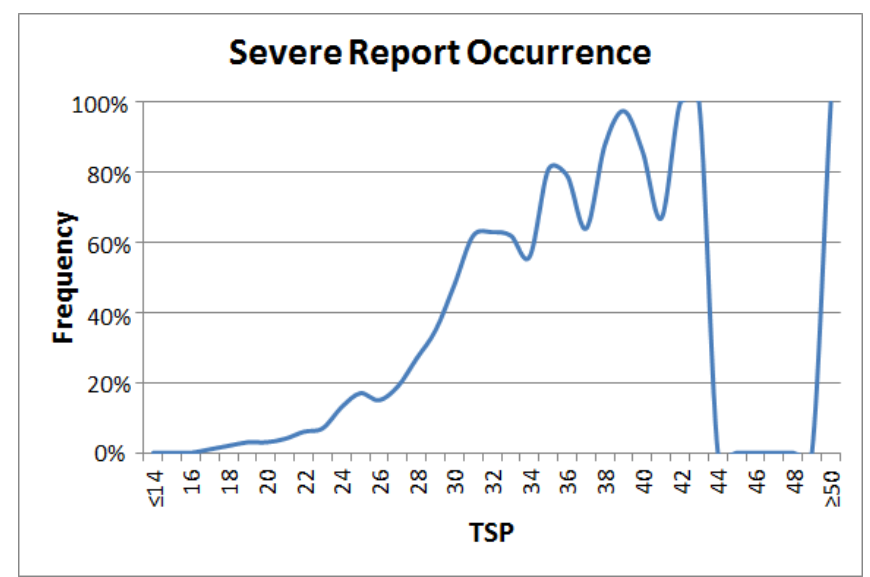

Figure 13. The distribution of reported severe weather frequency based on individual TSP values. The frequency of $0 \%$ between TSP values from 45 to 49 is likely due to a small number of days with those TSP and/or poor reporting of severe weather from largely unoccupied rural areas in east-central FL.

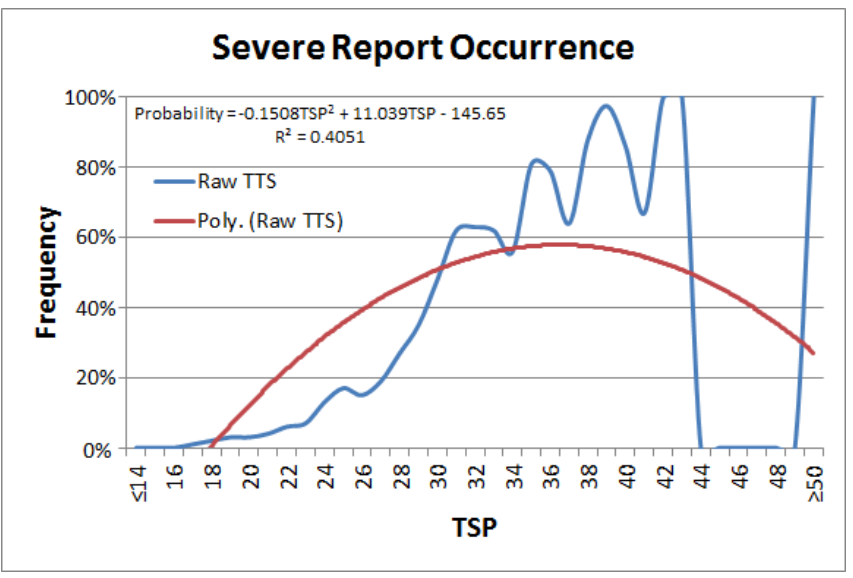

Figure 14. Same as Fig. 13 except with a second order polynomial curve (red line) fit to the TSP values (blue line).

distributions in Figs. 12 and 13 suggests a logistic curve response. Being a continuous function, the bestfit logistic curve also avoids the possible problems of categories - overly large bins to get sufficient sample size per bin or noisy inconsistent results from insufficient sample size per bin. Other possible problems from a categorical approach include inconsistent behavior of adjacent categories, such as a decreasing (increasing) likelihood of severe weather with increasing (decreasing) TSP. Similarly, a slight (large) change in TSP might result in a large (small) change in the likelihood of severe weather by crossing into a new category (staying within a category). Finally, the best-fit logistic curve provides extrapolation of the probability of severe weather that is consistent with the other data to slightly higher or slightly lower TSP not observed during the development POR.
The 45WS offered to assist the AMU by developing a best-fit logistic curve because the logistic curve is constrained to be within $0 \%$ to $100 \%$ and is often used in probabilistic regression. Fitting a logistic curve could not be solved analytically and had to be done iteratively (in this case manually) because of the lack of statistical software. Each of the three coefficients was stepwise iterated until the root-mean-square error of the differences between the logistic curve and the observed values was minimized to be $<0.0005$ (optimized to three decimal places). The best-fit logistic regression curve (Fig. 15) is specified as follows:

$$
\text { Freq. }=100 *\left(\frac{1}{1+\exp (-(0.764+0.270 *(T S P-34.013)))}\right) .
$$

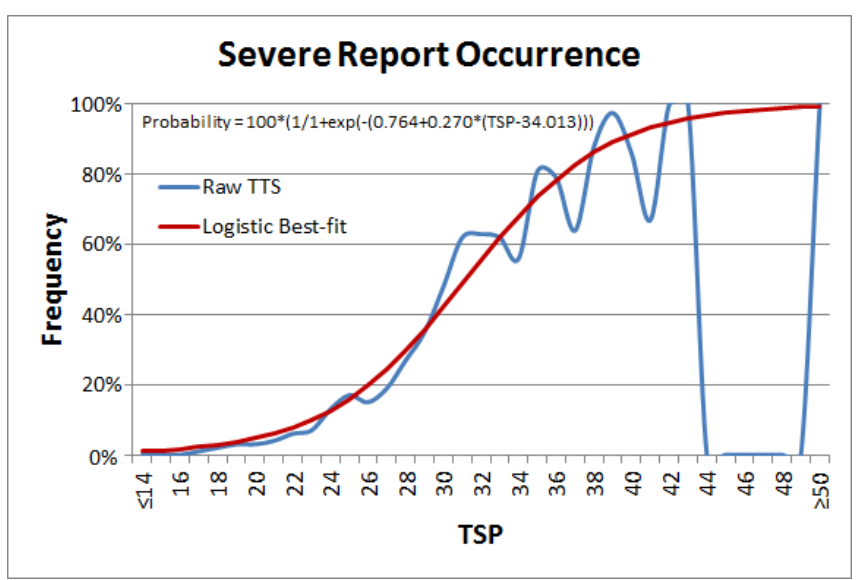

Figure 15. Same as Fig. 13 except with a best-fit logistic regression curve (red line) fit to the TSP values (blue line). A correlation coefficient (r) is not available because best-fit logistic curves must be done iteratively and manually. Bin-to-bin variations are smoothed over and gaps with anomalous results (TSP 4449) are bridged.

The 45WS also tested other best-fit curves (quadratic, exponential, and power law) for completeness in case they performed better. These three curves exceeded $100 \%$ at the higher TSP values, similar to the second-order polynomial curve. The logistic curve, however, is a better fit to the data than the other methods and offers the additional desired behavior of not being able to exceed $100 \%$ at large TSP values or falling below $0 \%$ at low TSP values. Table 2 shows the final TSP values and corresponding occurrences of reported severe weather based on the logistic regression curve shown in Fig. 15 that were implemented in the MIDDS GUI. The mean difference between the actual data and the logistic curve is -0.66 , indicating that the logistic curve has a slight tendency to over- 
Table 2. The final TSP values and corresponding occurrences of reported severe weather (Severe Freq) based on the logistic regression fit.

\begin{tabular}{|l|l|l|l|l|l|l|l|l|l|l|l|l|l|l|l|l|l|l|l|}
\hline TSP & $\mathbf{1 4}$ & $\mathbf{1 5}$ & $\mathbf{1 6}$ & $\mathbf{1 7}$ & $\mathbf{1 8}$ & $\mathbf{1 9}$ & $\mathbf{2 0}$ & $\mathbf{2 1}$ & $\mathbf{2 2}$ & $\mathbf{2 3}$ & $\mathbf{2 4}$ & $\mathbf{2 5}$ & $\mathbf{2 6}$ & $\mathbf{2 7}$ & $\mathbf{2 8}$ & $\mathbf{2 9}$ & $\mathbf{3 0}$ & $\mathbf{3 1}$ & \multicolumn{10}{|c|}{} \\
\hline $\begin{array}{l}\text { Severe } \\
\text { Freq }(\%)\end{array}$ & 1 & 1 & 2 & 2 & 3 & 4 & 5 & 6 & 8 & 10 & 13 & 16 & 20 & 24 & 30 & 36 & 42 & 49 \\
\hline
\end{tabular}

forecast - which is acceptable because this provides increased safety. The best-fit logistic curve offers an improvement of just over 19\% more than the original categorical approach, and that improvement is a higher probability of severe weather, which is conservatively safer. In addition, the logistic curve bridges gaps of TSP in the POR used in developing this tool and extends the technique to higher and lower values of TSP not covered by the categorical approach, covering 52\% more TSP than the categorical approach.

\section{c. Development for MIDDS}

The AMU developed the 1500 UTC SWT in MIDDS using the Tool Command Language and its associated Tool Kit (Tcl/Tk). The user starts the tool from the main weather menu on MIDDS. The program executes the $\mathrm{Tcl} / \mathrm{Tk}$ code to compute and retrieve sounding parameters and then presents the user with the GUI for manual input. Then the code computes a threat score for each parameter and the TSP for the sounding. The tool displays the output in two graphical windows for the user to view, and then saves two files in MIDDS for archive.

\section{1) THE GUI}

When the user executes the program in MIDDS, a message window is displayed notifying the user that the program is acquiring the sounding data and calculating the parameters. Once the sounding parameters are ready, the GUI is displayed for the user to enter information about the 200-hPa jet position and flow regime as shown in Fig. 16. In order to provide assistance, there is a Help button in the upper right of the GUI window that describes how to use the GUI and gives a description of the tool itself. Next, the date is displayed in two formats just above the questions on the left-year and day of the year, and calendar day in month/day/year format. The two gray buttons below the dates associated with each of the two questions provide a definition of each parameter via a pop-up window when the mouse is positioned over them. To help answer the questions, the user can click the white button at the right end of each row to display maps of the phenomenon being assessed. Once the user clicks one of the gray buttons, the choice is displayed in the box at the far right of the window. After both choices are made, the user clicks the green box in the lower left to calculate the TSP. The GUI then closes, and two other windows open with the results.

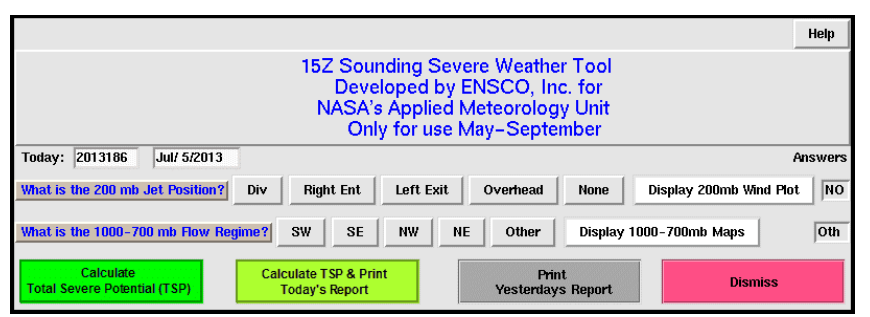

Figure 16. The 1500 UTC sounding-based SWT GUI.

$$
\begin{gathered}
\text { The Total Severe Potential (TSP) from the } \\
15: 00 \mathrm{Z} \text { XMR sounding on } \\
\text { Jul/22/2013 is } 28 \\
\text { based on a TSP range of } 13-50 .
\end{gathered}
$$

From this TSP of 28 , severe weather was reported $30 \%$ of the time in one or more of the six east-central Florida counties using data from this 1989-2012 climatology.

OK

Figure 17. TSP summary window displayed in MIDDS that provides a quick overview of the tool's output to the user.

\section{2) OUTPuT WINDOws}

The TSP, reported severe weather occurrence, and associated information are shown in two windows in MIDDS. The first, shown in Fig.17, provides the user with a summary of the output from the tool. The first group of text (black font) displays the current sounding's time and date, the TSP, and the dataset's range of TSP values. The second group of text (red font) restates the TSP from the current sounding, displays the frequency of occurrence of reported severe weather based on the TSP, and reminds the user that the dataset 
is based on reported severe weather in six east-central Florida counties and the POR was 1989-2012. The summary window was designed to give the user a quick look at the information output by the tool.

The second window displayed (Fig. 18) shows all of the sounding parameters and their values used to derive the TSP. The heading shows the month, day, and year, plus year and day of the sounding. Below the heading is a table showing the index or parameter in the first (left) column. The next four columns show the low, medium, high, and very high severe thresholds for each index or parameter to serve as a reference for the user. The last (right) column shows the value of the index or parameter from the sounding being evaluated.
The next section of text below the table displays the time of the sounding, the TSP, and the reported occurrence of severe weather based on the TSP. Finally, the paragraph at the bottom of the window reminds the forecaster that this tool should be used as a guide when determining the day's severe weather potential at $\mathrm{KSC} / \mathrm{CCAFS} / \mathrm{PAFB}$ as the TSP value is based on the climatology of severe weather occurrence in six east-central Florida counties. The forecaster must also consider the development and position of the sea breeze front and any outflow boundaries that could serve as triggers for convection and possibly lead to severe weather.

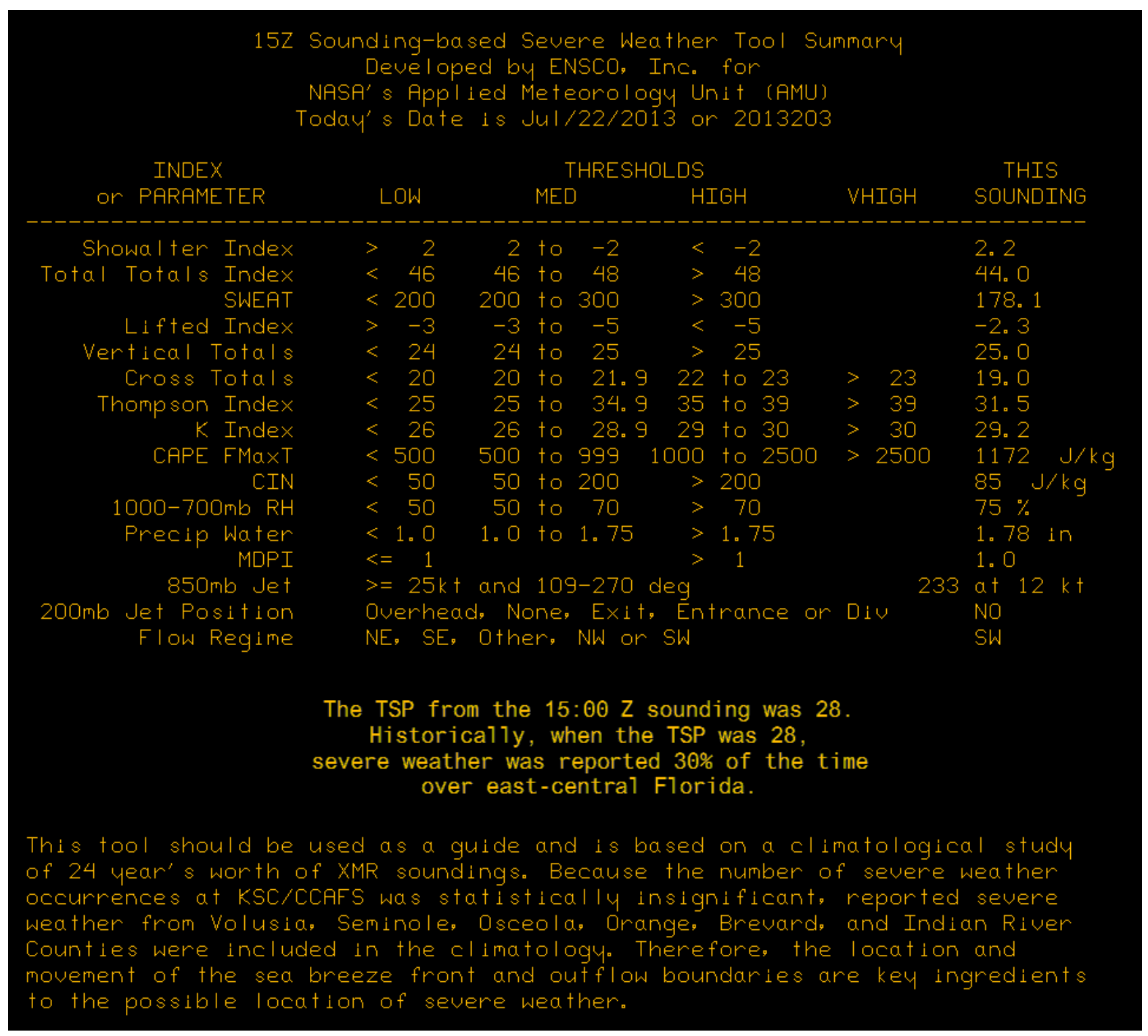

Figure 18. Detailed TSP window displayed in MIDDS provides the user with index and parameter severe thresholds and the specific values derived from the current sounding used to generate the TSP. 


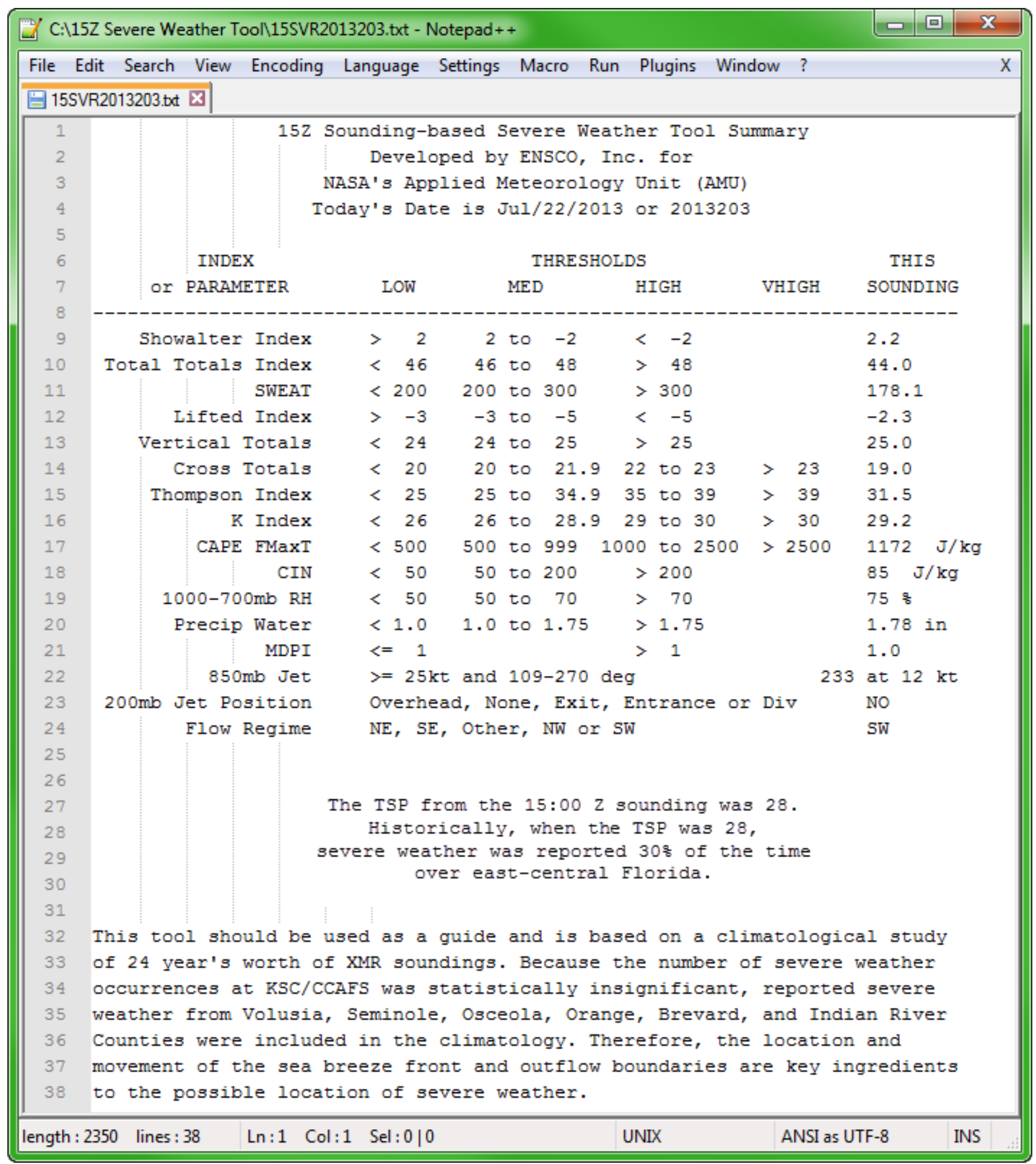

Figure 19. Sample output from a text file saved in MIDDS. The text file is identical to the output displayed in the detailed TSP window (Fig. 18).

In addition to the two output windows, the AMU code saves two files to MIDDS for archive purposes. One is a comma-separated value (CSV) formatted file that displays the month, day, and year, plus year and day of the sounding, plus the indices and parameters with their associated values from the sounding. The second file (Fig. 19) is saved in MIDDS as a text file that replicates the detailed TSP output window in Fig. 18 and can be displayed in any text viewer software. 


\section{3) TESTING AND TRAINING}

The AMU tested the tool by running it each day that a sounding was available to ensure MIDDS was calculating the correct values. Each parameter's threat score and resulting TSP were manually calculated to ensure they were identical to the corresponding threat scores calculated by the code in MIDDS for each sounding. To automate this process, the AMU wrote code in Microsoft Excel Visual Basic for Applications, which imported the MIDDS CSV files and calculated each parameter's threat score and the TSP to compare to the manually calculated values. The code was tested on 14 soundings to make sure it worked before discontinuing manual calculation of the threat scores and TSP. The AMU tested 40 soundings by comparing values from the MIDDS CSV files to the Excel-calculated values and ensuring consistent values before installing the software on the operational MIDDS.

In order to allow the forecasters to start using the tool during the current warm season, the AMU provided training to the 45WS during two of their daily weather discussions in mid-July 2013 to ensure all shift workers were present. For the presentations, the AMU gave a very short overview of the work and then demonstrated how to use the tool in MIDDS. Since that time, the tool has been used in daily operations.

\section{d. Other comments}

The 45WS found the 1500 UTC SWT much easier to use than its predecessor, had more consistent forecasts, and covered a wider range of TSP. As a result, they asked the AMU if similar changes could be retrofitted to the previously developed 1000 UTC tool. The reduction in subjective input and use of best-fit logistic curves were especially desired. Fortunately, the AMU was ahead of schedule on the 1500 UTC task and was able to make the changes, with the $45 \mathrm{WS}$ providing the best-fit logistic curve as with the 1500 UTC tool.

\section{Possible improvements}

There are several opportunities to improve this tool. Updating the tool to incorporate additional warm seasons would be useful because only $24 \mathrm{yr}$ of observations were available. This is especially important for severe weather, which has an inherently low frequency of occurrence. Other statistical techniques may allow better selection of the predictor variables and their thresholds. Further, accounting for lack of independence between predictor variables is especially impor- tant. In this regard, possible statistical techniques include discriminant analysis and/or canonical variance. Another approach would be to use principal component analysis to identify the combination of predictor variables that explain the most linear variance in decreasing order of the independent components, then perform the regression on using the components in decreasing order until insufficient new variance is explained. Also, alternative methods to predict the likelihood of severe weather should be considered, such as parameter combinations (e.g., logistic regression). Although logistic regression had not proved useful when developing the SWT based on the 1000 UTC sounding (Watson 2011), it should be reconsidered because $83 \%$ of the subjective predictor variables have been eliminated. Those subjective predictor variables may have introduced too much noise and precluded the logistic regression from working as well as it might. This logistic regression is different than the logistic curve fitting to the TSP described previously. The logistic regression would predict the probability of severe weather directly from the best sequential combination of predictor variables (in order of new variance explained), rather than create a logistic curve response to the TSP as done in this study. In addition, other approaches, such as classification and regression trees, may be applicable. Finally, it would be beneficial to investigate the gain in skill in predicting severe weather from the 1500 UTC sounding compared to the 1000 UTC sounding. Identifying the days when the 1500 UTC sounding will or will not be useful in improving the severe weather forecast could provide a cost savings by not releasing the 1500 UTC sounding when unnecessary.

\section{Conclusions}

Because people and property at KSC and CCAFS are at risk when severe weather occurs, the $45 \mathrm{WS}$ tasked the AMU to develop a warm-season SWT for use in MIDDS based on the late morning (1500 UTC) KXMR sounding. The 45WS daily and weekly severe weather forecasts are used by managers to determine if they need to limit an activity (such as working on gantries ) or protect property (such as a vehicle on a pad). The 45WS requested this SWT be based on the 1500 UTC sounding because they frequently make decisions to issue a severe weather watch and other severe weather warning support products in the late morning, and this sounding is more representative of the atmospheric instability than the early morning sounding. 
The AMU built upon their previous tasks in developing severe weather decision aids by using three existing datasets that were compiled during those tasks and updating them with 2011 and 2012 data. Those datasets included upper-level $(200 \mathrm{hPa})$ jet stream analyses, severe storm reports, and daily lightning flow regimes. The AMU developed two new datasets for this task that included the 1500 UTC KXMR soundings based on $24 \mathrm{yr}$ of warm-season months from 1989 to 2012 and the stability parameters derived from those soundings.

The AMU determined a threat score based on individual sounding stability indices and parameter thresholds and, from those, calculated a TSP for every 1500 UTC sounding in the $24-y r$ database. These TSP values then were compared to reported severe weather occurrences on each day with a sounding. As a result, a frequency of reported severe weather was determined for each TSP, a best-fit logistic regression curve was developed, and then the values were incorporated in a GUI for an operational tool.

The AMU delivered the updated SWT to the 45WS in mid-July 2013, and it is being used to support daily and launch operations. The tool automatically retrieves and calculates the required indices and parameters from the sounding and then presents the user with a GUI to choose the $200-\mathrm{hPa}$ jet position and 1000-700-hPa flow regime. One of the enhancements over the previous form of the GUI was the substantial reduction of the subjective questions posed to the forecasters, thereby streamlining the process of running the tool and creating a more objective assessment of the daily warm-season severe weather threat.

Acknowledgments. The authors thank the 45WS launch weather officers and forecasters, who provided valuable feedback on the methods used and the development of the GUI, and Mr. Stephen Madison of Computer Sciences Raytheon, who helped troubleshoot the AMU code and install the operational software on MIDDS.

\section{REFERENCES}

Ander, C. J., A. J. Frumkin, J. P. Koermer, and W. P. Roeder, 2009: Study of sea-breeze interactions which can produce strong warm-season convective winds in the Cape Canaveral area. Preprints, 16th Conf. on AirSea Interaction/Eighth Conf. on Coastal Atmospheric and Oceanic Prediction and Processes, Phoenix, AZ, Amer. Meteor. Soc., J8.3. [Available online at ams.confex.com/ams/pdfpapers/143062.pdf.]
Bauman, W. H., III, W. P. Roeder, R. A. Lafosse, D. W. Sharp, and F. J. Merceret, 2004: The Applied Meteorology Unit - Operational contributions to Spaceport Canaveral. Preprints, 11th Conf. on Aviation, Range, and Aerospace Meteorology/22nd Conf. on Severe Local Storms, Hyannis, MA, Amer. Meteor. Soc., 6.3. [Available online at ams.confex.com/ams/ pdfpapers/78114.pdf.]

, M. M. Wheeler, and D. A. Short, 2005: Severe weather forecast decision aid. NASA Contractor Report CR-2005-212563, Kennedy Space Center, FL, 50 pp. [Available online at science.ksc.nasa.gov/amu/finalreports/severe-tool-final.pdf.]

Lambert, W., and W. Roeder, 2008: Update to the lightning probability forecast equations at Kennedy Space Center/Cape Canaveral Air Force Station, Florida. Preprints, 20th International Lightning Detection Conference/Second International Lightning Meteorology Conference, Tucson, AZ, Roy. Meteor. Soc., 16 pp. [Available online at www.vaisala.com/en/events /ildcilmc/Documents/Update\%20to\%20the\%20Lightnin g\%20Probability\%20Forecast \%20Equations \%20at $\% 20$ Kennedy\%20Space\%20Center.PDF]

Lericos, T. P., H. E. Fuelberg, A. I. Watson, and R. L. Holle, 2002: Warm season lightning distributions over the Florida peninsula as related to synoptic patterns. Wea. Forecasting, 17, 83-98, CrossRef.

Madura, J. T., W. H. Bauman III, F. J. Merceret, W. P. Roeder, F. C. Brody, and B. C. Hagemeyer, 2011: The Applied Meteorology Unit: Nineteen years successfully transitioning research into operations for America's space program. Preprints, Conf. on Transition of Research into Operations/27th Conference on Interactive Information Processing Systems (IIPS), Seattle, WA, Amer. Meteor. Soc., J10.2. [Available online at ntrs.nasa.gov/archive/nasa/casi.ntrs.nasa.gov/20110007 021.pdf.]

Space Science and Engineering Center, 2012: McIDAS-X User's Guide Version 2012.2. University of Wisconsin, Madison, WI, 591 pp. [Available online at ssec.wisc. edu/mcidas/doc/users_guide/2012.2/users guide.pdf.]

Uccellini, L. W., and D. R. Johnson, 1979: The coupling of upper and lower tropospheric jet streaks and implications for the development of severe convective storms. Mon. Wea. Rev., 107, 682-703, CrossRef.

Watson, L., 2011: Upgrade summer severe weather tool. NASA Contractor Report CR-2011-216299, Kennedy Space Center, FL, 31 pp. [Available online at science.ksc.nasa.gov/amu/final-reports/severe-toolupgrade-II.pdf.]

Wheeler, M. M., and W. P. Roeder, 1996: Forecasting wet microburst on the central Florida Atlantic Coast in support of the United States space program, Preprints, 18th Conf. on Severe Local Storms, San Francisco, CA, Amer. Meteor. Soc., 654-658. 
2009: Severe weather and weak waterspout checklist in MIDDS. NASA Contractor Report CR-2009-214760, Kennedy Space Center, FL, 16 pp. [Available online at science.ksc.nasa.gov/amu/final-reports/svr-wX-wkshtmidds.pdf.]
2010: Upgrade summer severe weather tool in MIDDS. NASA Contractor Report CR-2010-216282, Kennedy Space Center, FL, 19 pp. [Available online at science.ksc.nasa.gov/amu/final-reports/severe-toolupgrade.pdf.] 